University of Nebraska - Lincoln

DigitalCommons@University of Nebraska - Lincoln

\title{
Digital Analysis of Leaf Surface Area: Effects of Shape, Resolution, and Size
}

Jeffrey Bradshaw

University of Nebraska-Lincoln, jbradshaw2@unl.edu

Marlin E. Rice

lowa State University

John W. Hill

lowa State University

Follow this and additional works at: https://digitalcommons.unl.edu/panhandleresext

Part of the Agriculture Commons

Bradshaw, Jeffrey; Rice, Marlin E.; and Hill, John W., "Digital Analysis of Leaf Surface Area: Effects of Shape, Resolution, and Size" (2007). Panhandle Research and Extension Center. 44.

https://digitalcommons.unl.edu/panhandleresext/44

This Article is brought to you for free and open access by the Agricultural Research Division of IANR at DigitalCommons@University of Nebraska - Lincoln. It has been accepted for inclusion in Panhandle Research and Extension Center by an authorized administrator of DigitalCommons@University of Nebraska - Lincoln. 


\title{
Digital Analysis of Leaf Surface Area: Effects of Shape, Resolution, and Size
}

\author{
Jeffrey D. Bradshaw, ${ }^{1 *}$ Marlin E. Rice, ${ }^{1}$ and John H. Hill ${ }^{2}$
}

\begin{abstract}
The effects of shape, size, and capture resolution on digital area measurement were investigated to accurately and precisely estimate leaf surface area. A digital scanner was used to measure two simple shapes (circle and square) at three resolutions $(118.159,236.270$ and 472.441 pixels $/ \mathrm{cm}$ ) and five sizes $\left(3.14,12.58,28.29,50.29\right.$, and $\left.78.60 \mathrm{~cm}^{2}\right)$. Additionally, the accuracy and precision of two digital scanner models were compared using two shapes (circle and square) of similar size at five resolutions $(29.528,39.370,59.055,118.159,236.270$, and 472.440 pixels $/ \mathrm{cm}$ ). A method is described to measure leaf area using an image histogram and photographic software tools (Photoshop ${ }^{\circledR}$ ). This method was validated by comparison of the digitally captured images to a leaf area meter (LI-COR $\left.{ }^{\circledR} 3100\right)$. Overall, simple changes in shape have a statistically significant effect on the accuracy of digital measurements of area for some sizes and resolutions.

KEY WORDS: digital analysis, digital scanner, leaf area measurement, insect herbivory
\end{abstract}

As early as 1928, photoelectric devices were used to measure leaf area (Květ and Marshall, 1971). With devices such as the photoelectric planimeter, leaf area could be quantified with a level of precision between 4-100 $\mathrm{mm}^{2}$ depending on a number of settings or modifications (Květ and Marshall, 1971). Then, with computers and more sophisticated light-scanning technology of the 1970's, electronic leaf area meters allowed measurements as small as $1 \mathrm{~mm}^{2}$ (Kogan and Turnipseed, 1980) and apparently $0.1 \mathrm{~mm}^{2}$ (LI-COR ${ }^{\circledR}$ Incorporated 2004). The leaf area meter is still used as the standard for validating new tools and techniques for measuring leaf area (Bowers et al., 1999; O’Neal et al., 2002).

Tools and techniques for analyzing digital images are common in science. Various companies and organizations advertising digital software claim to measure many facets of a digital image (Russ, 2004; National Institutes of Health, 2007). Additionally, with computer programs that allow batch-processing, large sample sizes could be analyzed efficiently. O'Neal et al. (2002) demonstrated that an inexpensive, flatbed scanner is an accurate and precise tool for measuring leaf area and herbivory. However, they did not determine the most accurate and precise resolution for image capture. Additionally, the effects of varying shapes and surface areas on accuracy and precision were not described. We adapted a technique, similar to O'Neal et al. (2002), for use with an image histogram. Using this technique, we determined the effects of shape, size, and image resolution for image analysis and data acquisition, the consistency of the effects of shape and resolution between two digital scanners, and the relative relationship between our described technique and a leaf area meter in measuring leaf area.

\footnotetext{
${ }^{1}$ Department of Entomology, Iowa State University, Ames, Iowa 50011.

2 Department of Plant Pathology, Iowa State University, Ames, Iowa 50011.

${ }^{3}$ Corresponding author: Iowa State University, Department of Entomology, Ames, Iowa 50011, e-mail: bradshaw@iastate.edu
} 


\section{Materials and Methods}

For all experiments in this study, we used Adobe ${ }^{\circledR}$ Photoshop ${ }^{\circledR}$ (Adobe Systems Incorporated, 2002) to record repetitive imaging tasks. This was accomplished by using a GUI (graphical user interface)-based scripting program. Adobe Photoshop terms these scripted programs "actions". Such scripting or actions allows a series of repetitive tasks to be recorded and can be run on multiple image files via "automation" or batch processing. To count pixels in digital images an action was created to automatically open an image file, display the image histogram (using the "Histogram" function), wait until commanded to exit the histogram (i.e., press enter), and close the file. This action was combined with a batch process that we created, that would serially apply the action to all of the files in a directory. The total pixel count is displayed in the histogram window, labeled "Pixels", and the number of black pixels can be determined by resting the computer cursor over the histogram at level 0 and recording the number of pixels (labeled as "Count" within the histogram window). For more intuitive comparisons, digitally scanned areas (in pixels) were converted to square centimeters by dividing the number of black pixels into the number of squared total pixels $/ \mathrm{cm}$ in the images as determined by Photoshop, i.e.,

$$
\operatorname{Area}\left(\mathrm{cm}^{2}\right)=\frac{\text { black pixels }}{(\text { total pixels } / \mathrm{cm})^{2}}
$$

\section{Experiment 1}

To determine the influence of shape, size, and resolution on scanner accuracy (i.e., measured area minus expected area) and precision (i.e., standard error of the mean of the measured area) we used two uniform shapes (circle and square) and scanned them with a digital scanner, Hewlett-Packard ${ }^{\text {TM }}$ Scanjet 4670 (Hewlett-Packard Company, L.P., Palo Alto, Calif.). Images were captured with 1 bit per pixel. For each shape five sizes were compared $\left(3.14,12.58,28.29,50.29\right.$, and $78.60 \mathrm{~cm}^{2}$ ). Shapes were constructed using Photoshop with 472.441 pixels $/ \mathrm{cm}$ and printed with 472.441 pixels/cm on a Hewlett-Packard Laserjet $4000 \mathrm{TN}$ printer on white paper. One printed image was produced for each shape and size. The printed images were placed image side down on the scanning surface, held in place by a 8.5 by 11 -in. pane of glass, captured from arbitrary locations $(n=8)$ and rotated at arbitrary angles relative to the image-capturing sensor. Printed images were captured at bit depth of 1 bit/pixel (black and white).

We hypothesized that the approach angle of the scanner head to the object being scanned would affect the results of the calculated area of that object. Therefore, two shapes were chosen for two reasons: First, circles have a uniform approach angle relative to the scanner's image-capturing sensor; therefore, this shape should have an error only associated with the scanner and not due to changes in perimeter morphology within a given size if placed at random locations upon the scanning plane. Second, squares do not have a uniform approach angle relative to the scanner's image-capture sensor; therefore, a square should have error attributable to the scanner and perimeter morphology within a given size. Thus, each shape was printed only once (to minimize printer error) but its position and planar orientation were adjusted before each replicate scan. The effects of shape and size were tested for three digital resolutions, 118.159, 236.270, and 472.440 pixels/cm. 


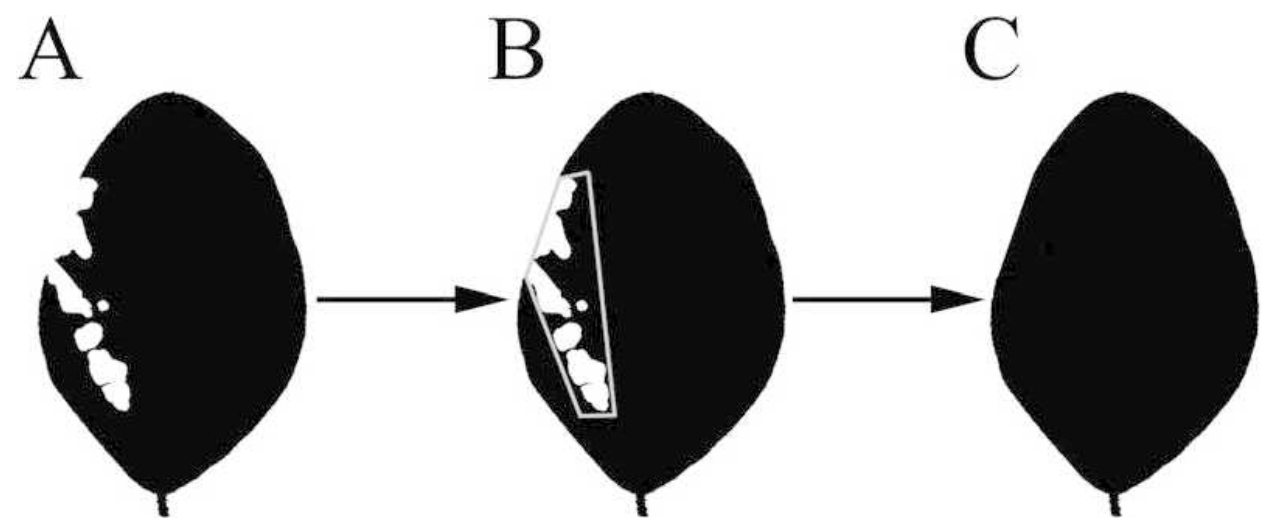

Fig. 1. Process by which leaf area can be interpolated from an injured leaf. An area of an injured leaf (A) is selected (B) with the "Polygonal Lasso tool" in Adobe ${ }^{\circledR}$ Photoshop ${ }^{\circledR}$, including the injured leaf margin as shown, and the pixel levels reduced to zero to interpolate the leaf area (C) prior to injury.

\section{Experiment 2}

To test for consistency between digital scanners, two scanners (Hewlett-Packard Scanjet 4670 and 6300) were compared using a $20.25-\mathrm{cm}^{2}$ square and a $20.17-\mathrm{cm}^{2}$ circle. These devices were chosen for three reasons: First, to simplify our choice among the many brands. Second, we had access to these models. Third, an assumption was made that manufactured elements may be more similar within a brand than between brands. Both shapes $(\mathrm{n}=8)$ were scanned at six resolutions; $472.440,236.270,118.159,59.055,39.370$, and 29.528 pixels/cm (i.e., $1200,600,300,150,100,75$ pixels/in). Image capture was confined to the lower left corner of the scanner bed, otherwise image capture method was as described for experiment 1 .

\section{Experiment 3}

For this experiment a method was developed for calculating leaf herbivory of whole leaves. The method is similar to that used by Kogan and Turnipseed (1980); however, here it is adapted for use on the PC.

Adobe Photoshop was used to create estimates of leaflet area of soybean, Glycine $\max ($ L.) (Fabaceae), prior to herbivory by the bean leaf beetle, Ceratoma trifurcata (Förster) (Coleoptera: Chrysomelidae), using injured leaflets as follows (Fig. 1A-C). After all leaflets were digitally scanned (at $1 \mathrm{bit} / \mathrm{pixel}$ ), and the resulting images saved, an action was created that would open an image of an injured leaflet (Fig. 1A). The user was then prompted to select an area, using the "Polygonal Lasso Tool" (Fig. 1B), surrounding the injured area of the leaflet and then to select the "OK" button to proceed to the next action step. Photoshop then ran the "levels" adjustment function and reduced the tonal range of the selected area to 0 (using the "levels" adjustment function); thereby converting white pixels to black (Fig. 1C). The adjusted image was then saved to a new file as an uncompressed, tagged image file format (TIFF). We refer to these adjusted, leaflet images as the interpolated leaflet area (ILA). 
The process described above allowed us to approximately reproduce the area of the leaflet prior to beetle herbivory (see Fig. 1A-C). Where there was herbivory of the leaflet margin a straight-line selection was drawn across the marginal gap (as illustrated in Fig. 1B) to facilitate a conservative estimation of consumed leaflet area.

A stationary leaf area meter (LI-COR LI-3100, Lincoln, NE) was used to validate the above method and the use of a digital scanner (Hewlett-Packard ${ }^{\mathrm{TM}}$ Scanjet 4670) for measuring soybean leaf herbivory. Soybean, var. Clark, leaflets $(n=22)$ were exposed to bean leaf beetle feeding in closed $100 \times 15-\mathrm{mm}$ Petri dishes with one beetle and leaflet per dish. Petri dishes were sealed with black electrical tape to prevent leaf desiccation and maintained at 16:8 L:D at approximately $23.3^{\circ} \mathrm{C}$. After 24 hours, leaflets were removed and pressed until dry (to reduce error due to leaflet morphology and thickness attributed to moisture content). Leaflets then were scanned using the leaf area meter and digital scanner. Images of digitally-scanned leaflets were uploaded to a computer (Dell ${ }^{\mathrm{TM}}$ OptiPlex GX150 with a Pentium ${ }^{\circledR} 6$ processor, Dell USA, Austin, Tex.), captured in black and white (i.e., 1 bit/pixel) and saved as an uncompressed TIFF file (as previously described for batch processing). The ILA (Fig. 1C), area of the injured leaflet (Fig. 1A) and the amount of consumed leaflet area (the different between the injured leaflet area and the ILA) was determined. For each image the "Histogram" function was used to measure the number of black pixels in the scanned image (all pixels at level 0 ).

The LI-COR 3100 was calibrated using a $50 \mathrm{~cm}^{2}$ metal disk according to manufacture recommendation for the LI-COR 3100 (LI-COR Incorporated 2004). The LI-COR 3100 has two resolution modes, $1 \mathrm{~mm}^{2}$ and $0.1 \mathrm{~mm}^{2}$. We operated the scanner at the $1 \mathrm{~mm}^{2}$ resolution mode as the model that was available to us was not equipped properly for use at a $0.1 \mathrm{~mm}^{2}$-resolution. Leaflets were scanned as described by Kogan and Turnipseed (1980). Injured soybean leaflets $(n=22)$, and printed, cutout copies of their corresponding ILA (printed at 472.441 pixels/cm) were placed on the center of the rotating belt of the meter and the output recorded. Injured leaflet area, ILA, and consumed leaflet estimates were compared by regression analysis between the LI-COR 3100 and digital scanner for three scanned resolutions $(118.159,236.270$, and 472.440 pixels $/ \mathrm{cm})$.

\section{Statistical analysis}

All experiments were conducted and analyzed as randomized designs. Proc Mixed was used for factorial analyses (SAS Institute, 2004a) and regression analysis was completed using the JMP statistical package (SAS Institute, 2004b). Based on the observed by predicted plot of residuals, each level of the factor "size" had a different variance in experiment 1 . Therefore, size was declared as both a continuous and a class variable. The continuous variable was used for covariance estimation. The class variable was used for means comparison in the factorial analysis of shape by resolution by size. Similarly, based on the observed by predicted plot of residuals, each level of the factor "resolution" had a different variance in experiment 2 . The continuous variable was used for covariance estimation. The class variable was used for means comparison in the factorial analysis of resolution by shape by scanner. A variance components model was used to describe the covariance structure in the data for experiments 1 and 2. Studentized residuals were examined for outliers in the factorial and regression analyses. In this study, bias is defined as the observed value minus a defined standard value. 


\section{Results}

\section{Experiment 1}

An object's shape, size, and the scanned resolution can affect the accuracy and precision of the measurement of area for some sizes and resolutions $(F=6.66$; $\mathrm{df}=$ 8,$210 ; P<0.0001$ ) (Table 1). In general there is an increase in bias and loss in precision as size increases for the highest resolution. Additionally, the lowest resolution tended to underestimate area while, conversely, the highest resolution overestimated area. The highest resolution, 472.441 pixels $/ \mathrm{cm}$, recorded the smallest $(-0.002 \pm 0.002)$ and largest $(0.548 \pm 0.021)$ bias, for $3.14-\mathrm{cm}^{2}$ circles and $78.6-\mathrm{cm}^{2}$ squares, respectively (Table 1). According to comparisons of three-way interactions (shape*resolution*size) the highest resolution had the least stable bias between sizes within any one shape (Table 1). However, the bias of the highest resolution was statistically similar within, but not between, the largest size classes, 50.28 and $78.6 \mathrm{~cm}^{2}$ of the two shapes.

The bias of the two lowest resolutions, 118.159 and $236.27 \mathrm{~cm}^{2}$, were statistically similar for all sizes and shapes except for the largest size, $78.6 \mathrm{~cm}^{2}$ (Table 1). Similar to the highest resolution, the bias was statistically similar within, but not necessarily between the largest size classes (Table 1).

\section{Experiment 2}

Overall, the HP $4670\left(\overline{\mathrm{X}}\right.$ bias $\left.=0.005 \pm 0.001 \mathrm{~cm}^{2}\right)$ had significantly less bias $(F$ $=3074.15, \mathrm{df}=1,168, P<0.0001)$ than the HP $6300(\overline{\mathrm{X}}$ bias $=-0.085 \pm$ $0.001 \mathrm{~cm}^{2}$ ) for shapes of approximately $20 \mathrm{~cm}^{2}$. Additionally, the HP 4670 overestimated for both shapes at the lowest resolutions (29.528 and 39.37 pixels/ $\mathrm{cm}$ ) and the square shape at 59.055 pixels/cm (Table 2) while the HP 6300 underestimated area for all resolutions and shapes (Table 3). If shapes are combined, the HP 4670 had its lowest bias at 59.055 pixels $/ \mathrm{cm}\left(\overline{\mathrm{X}}\right.$ bias $=0.001 \pm 0.003 \mathrm{~cm}^{2}$ ) and at 39.37 pixels $/ \mathrm{cm}$ ( $\bar{X}$ bias $=0.052 \pm 0.003 \mathrm{~cm}^{2}$ ) for the HP 6300 . However, in this experiment these "best resolutions" are for one size only and did not incorporate the error attributed to varying the size of the scanned object.

\section{Experiment 3}

Soybean leaflets (either ILA or injured leaflets) scanned with a leaf area meter explain approximately $94 \%$ of the variation in digitally scanned leaflets regardless if scanned at 118.159, 236.270, and 472.440 pixels/cm (Figs. 2 and 3). The difference between the ILA and injured leaflets revealed that about $3 \%$ of variation in leaf consumption is left unexplained (Fig. 4) regardless of capture resolution. This assay had many leaflets with a small amount of feeding; therefore, the leaf area meter would be expected to be less accurate than a leaflet measured by digital scanner (O'Neal et al. 2002). That is in O'Neal et al. (2002), a leaf area meter overestimated simulated herbivory of $\leq 5.2 \mathrm{~cm}^{2}$ relative to a digital scanner. This is partly supported by our data for which bean leaf beetle herbivory was mostly overestimated by the LI-COR 3100 relative to a digital scanner for areas $\leq 0.5 \mathrm{~cm}^{2}$ (Fig. 4). Additionally, the LI-COR 3100 and 3000 series are known to have a larger margin of error for small areas, as much as $\pm 6-10 \%$ for areas of $1 \mathrm{~cm}^{2}$ or smaller with the error rate increasing for "complex shapes" for the LI-COR 3100C (LI-COR Incorporated, 2004). 


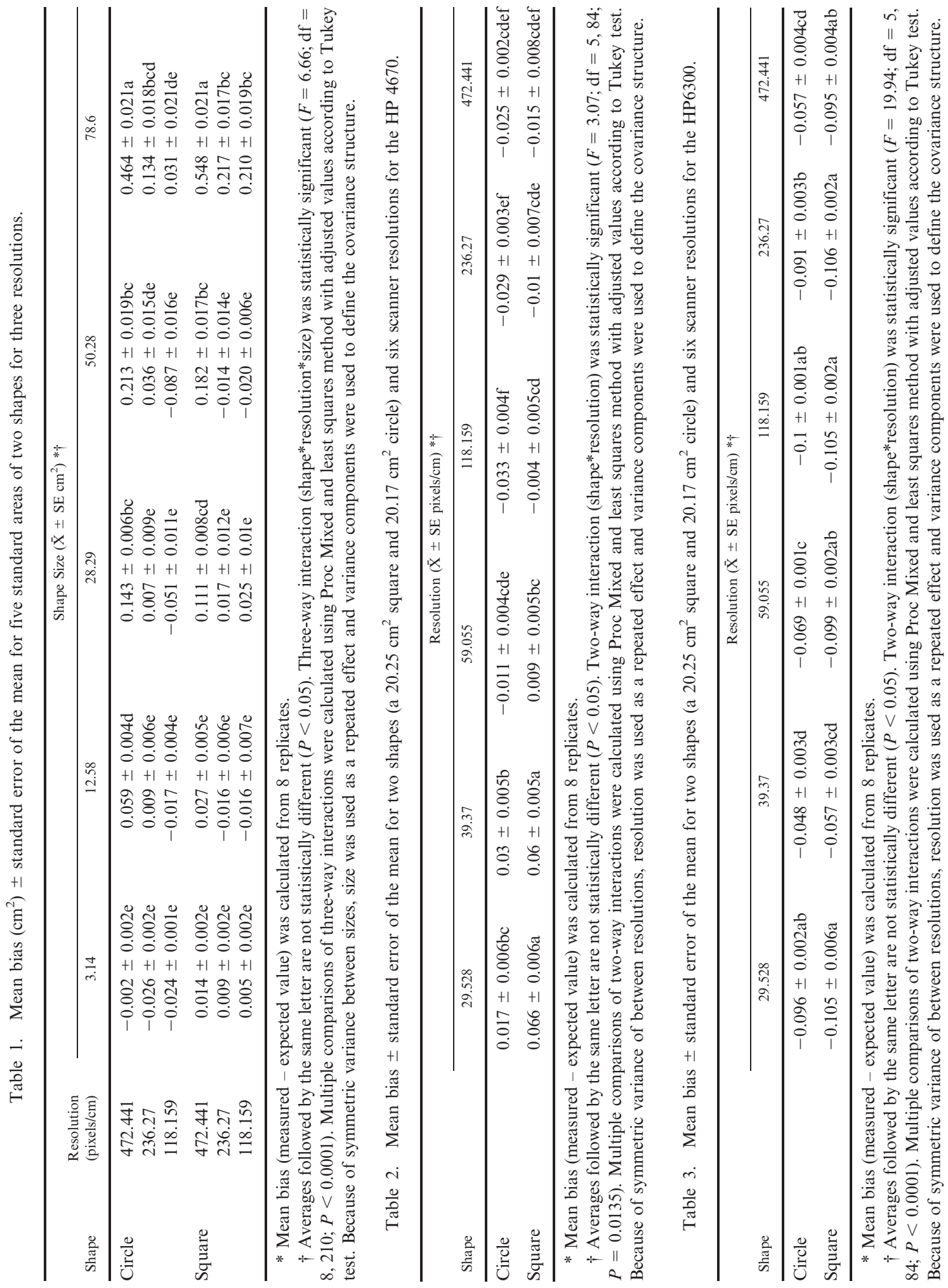




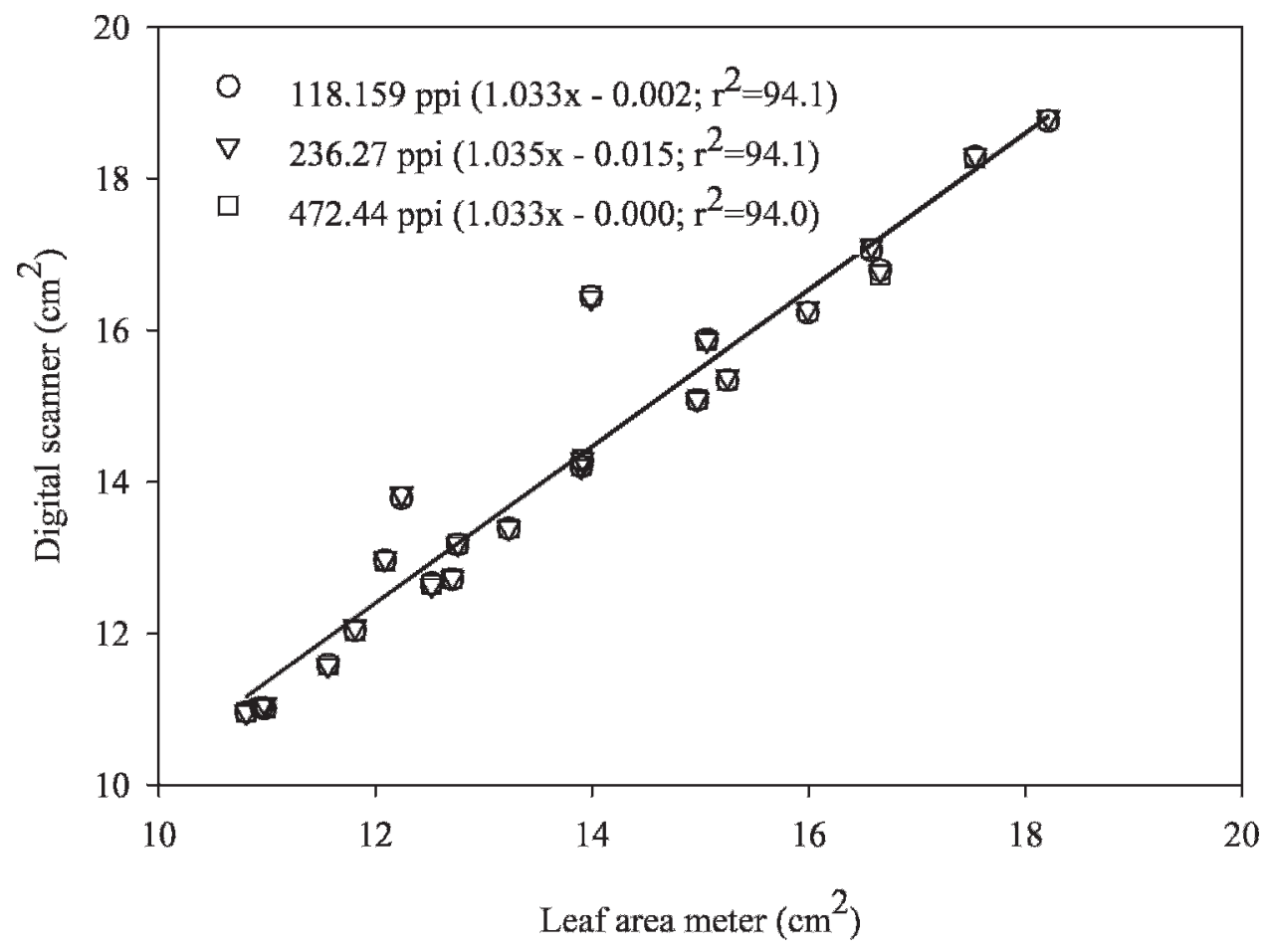

Fig. 2. Regression of interpolated soybean leaflet area as measured by digital scanner (HP 4670), at $118.159(F=316.22 ; \mathrm{df}=1,21 ; P<0.0001) 236.27(F=319.75 ; \mathrm{df}=1,21 ; P<0.0001)$, and $472.44(F=$ 310.6 ; df $=1,21 ; P<0.0001)$ pixels/cm, on leaf area meter (LiCor 3100$)$.

\section{Discussion}

These data indicate that using resolutions of either 118.159 or 236.27 pixels/cm (300 or 600 pixels/in, respectively) is sufficient for area measurement by digital scanner. These resolutions are accurate and precise for a variety of sizes and are relatively unaffected by slight changes in an object's geometry relative to higher resolutions, such as 472.441 pixels/cm (1200 pixels/in). When comparing three scan resolutions that have a similar bias for circles and squares $(118.159,236.27$, and 472.441 pixels $/ \mathrm{cm}$ [Tables 2 and 3]) significant differences are found when the shape sizes change (Table 1). However, at least for the HP 4670, some resolutions (e.g., 118.159 and 236.27 pixels/ $\mathrm{cm}$ ) are largely unaffected by changes in shape and size (i.e., areas between 3.14 and $\left.50.28 \mathrm{~cm}^{2}\right)$. For very small objects $\left(\leq 3.14 \mathrm{~cm}^{2}\right)$ it may be acceptable to use higher resolutions (i.e., 472.441 pixels $/ \mathrm{cm}$ ) especially for shapes that do not vary much in size. However, for example, experiments involving different plants with widely varying leaf sizes may suffer from random area-measurement errors.

The digital scanners compared in this study differ in one key component; maximum optical density. The image-scanning device for the HP 4670 (944.882 maximum pixels $/ \mathrm{cm}$ ) could be roughly four times more sensitive as the HP 6300 (236.27 maximum pixels/cm). The technology that enables this higher optical density may explain the difference in the area-measurement accuracy between these two scanners. Additionally, the distance between the image capturing sensor and the scanned object may vary between these two scanners. However, both of these are 


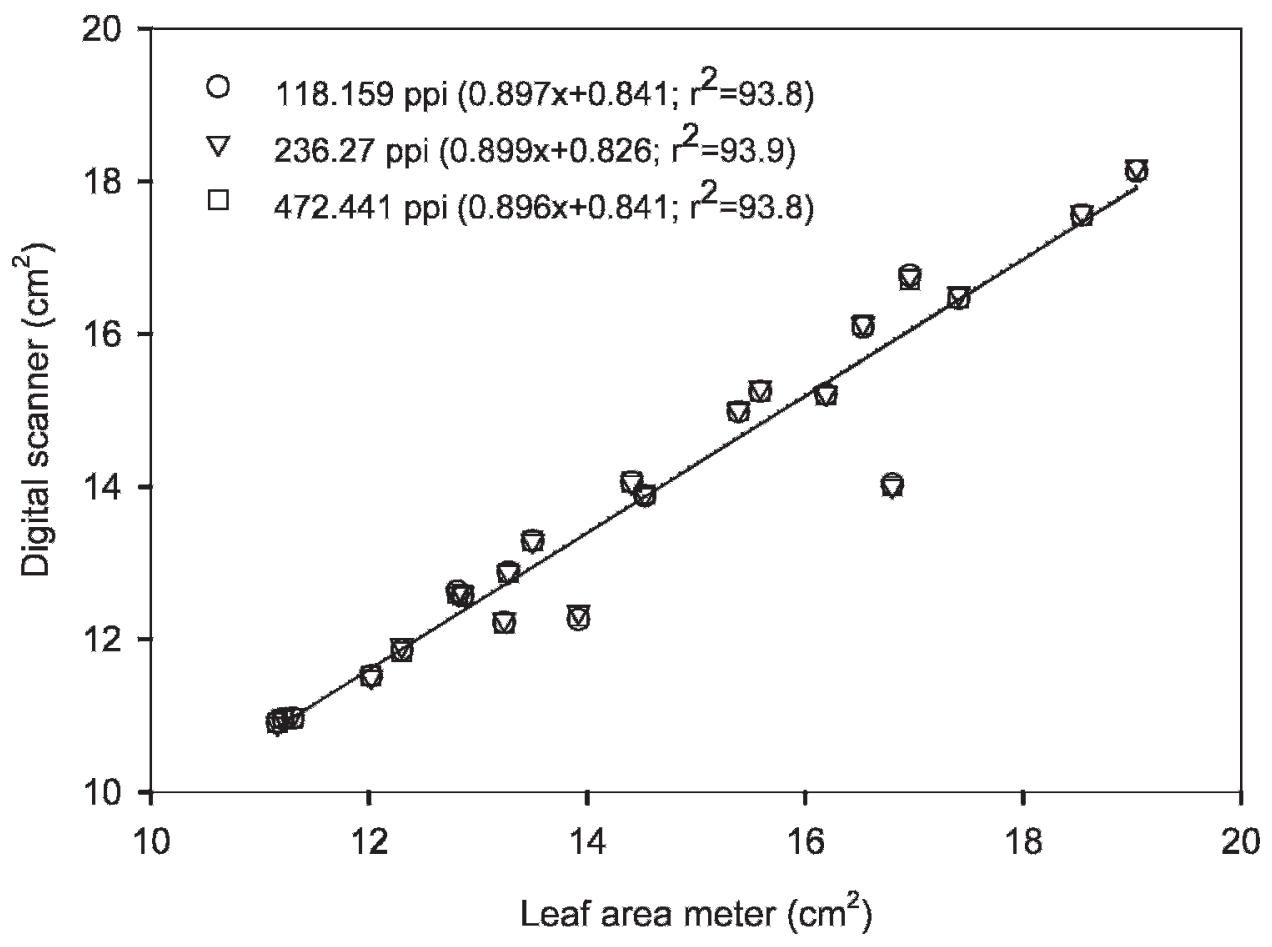

Fig. 3. Regression of injured-soybean leaflet area as measured by digital scanner (HP 4670), at 118.159 $(F=301.33 ; \mathrm{df}=1,21 ; P<0.0001) 236.27(F=305.16 ; \mathrm{df}=1,21 ; P<0.0001)$, and $472.44(F=303.74$; $\mathrm{df}=1,21 ; P<0.0001)$ pixels $/ \mathrm{cm}$, on leaf area meter (LiCor 3100$)$.

systematic errors and would not adversely affect measurement if confined to one scanner model.

The described technique for measuring leaf area produces measurements that are similar to the LI-COR leaf area meter. As described by O'Neal et al. (2002) the digital scanner is an accurate and precise tool for measuring leaf area and this is true for several scanner resolutions (Figs. 2, 3, and 4). Therefore, the above-described errors apparently are very similar between the two devices. However, according to LI-COR Incorporated (2004), measuring "complex shapes" (e.g., roots) under the highest resolution on the LI-COR $3100\left(0.1 \mathrm{~cm}^{2}\right)$ will result in error approximately $5 \%$ higher than "normal leaves". Because of the significant linear relationship between digital scanners and the LI-COR, it is likely that this error may afflict both. In fact both the leaf area meter and the digital scanners used in this study rely on the same basic technology, a linear sensor, to scan an object (some digital scanners use a CCD or charge-coupled device).

These results may help guide the selection of leaf shapes, sizes, and measurement tools for laboratory assays that challenge foliar pests with standard (e.g., lethal dose assays) or non-standard (e.g., assays involving multiple plant-species) leaf sections.

\section{Acknowledgements}

We thank Jessica L. Chisham, Department of Statistics, Iowa State University for assistance with statistical analysis. This study was supported, in part, by the North 


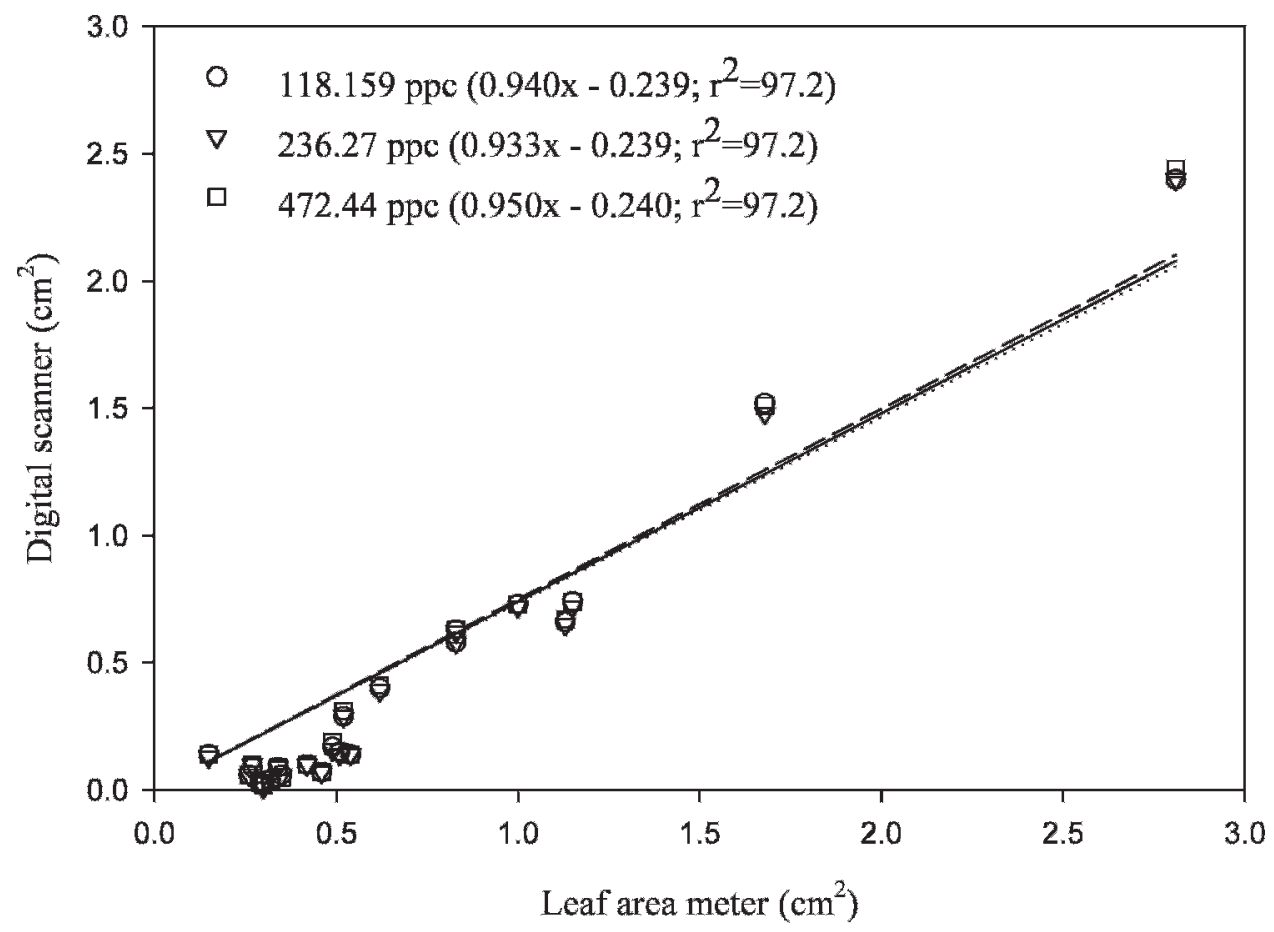

Fig. 4. Regression of soybean leaflet area consumed as measured by digital scanner (HP 4670), at $118.159(F=681.56 ; \mathrm{df}=1,21 ; P<0.0001) 236.27(F=702.95 ; \mathrm{df}=1,21 ; P<0.0001)$, and $472.44(F=$ 694.74; df $=1,21 ; P<0.0001)$ pixels $/ \mathrm{cm}$, on leaf area meter (LiCor 3100).

Central Soybean Research Program and the Iowa Soybean Promotion Board. Mention of a proprietary product does not constitute an endorsement by the authors or Iowa State University.

\section{Literature Cited}

Adobe Systems Incorporated. 2002. Adobe ${ }^{\circledR}$ Photoshop ${ }^{\circledR}$, Release 7.0.1. Adobe Systems Incorporated, San Jose, CA.

Bowers, G. R., M. M. Kenty, M. O. Way, J. E. Funderburk, and J. R. Strayer. 1999. Comparison of three methods for estimating defoliation in soybean breeding programs. Agronomy Journal 91:242-247.

Kogan, M., and S. G. Turnipseed. 1980. Soybean growth and assessment of damage by arthropods. In M. Kogan and D. C. Herzog (eds.). Sampling methods in soybean entomology, pp. 5-29. SpringerVerlag, New York. 587 pp.

Květ, J., and J. K. Marshall. 1971. Assessment of leaf area and other assimilating plant surfaces. In Z. Šesták, J. Čatský, and P. G. Jarvis (eds.). Plant photosynthetic prodution: manual of methods, pp. 517-555. Dr. W. Junk, The Hague. 818 pp.

LI-COR Incorporated. 2004. LI-3100C Area meter instruction manual. Lincoln, NE.

National Institutes of Health. 2007. NIH Image Manual (V1.61). Online: http://rsb.info.nih.gov/nih-image/ manuallcontents.html [Last accessed: 30 May 2007].

O’Neal, M. E., D. A. Landis, and R. Isaacs. An inexpensive, accurate method for measuring leaf area and defoliation through digital image analysis. Journal of Economic Entomology 95:1190-1194.

Russ, J. 2004. The Image analysis cookbook, version 6. Online: http://www.reindeergraphics.com/tutorial/ index.shtml [Last accessed: 30 May 2007].

SAS Institute. 2004a. JMP User Guide, Release 5. SAS Institute, Cary, NC.

SAS Institute. 2004b. SAS Help and Documentation, version 9.1.3. SAS Institute, Cary, NC. 